\title{
The Application of TCM Thought of Preventive Treatment of Disease in Clinical Practice
}

\author{
Huan $\mathrm{Li}^{1,2}$
}

\author{
${ }^{1}$ Henan Province Hospital of Traditional Chinese Medicine, 450002.Zhengzhou, 450000, Henan, P. \\ R. China \\ ${ }^{2}$ The Second Affiliated Hospital of Henan University of Traditional Chinese Medicine, 450000, \\ Henan, P. R. China
}

Keywords: Treatment of disease; Chinese medicine theory; Theoretical research; Clinical application

\begin{abstract}
The idea of preventive treatment of disease in traditional Chinese medicine (TCM) is to prevent the occurrence and development of diseases, prevent precautionary thoughts, that is, to prevent the occurrence of diseases before they are ill, to prevent the transmission of diseases after they have been sick. Prevention first, this article discuss the treatment of disease in the oncology disease, cardiovascular disease, respiratory system, digestive system disease in the clinical application, elaborated clinical advantage.

The theory of Preventive Treatment of Disease is an important feature and advantage of medicine in motherland. This doctrine has created a unique understanding of Chinese medicine in this area and insightful insights, is currently one of the hot spots in the medical profession at home and abroad. As early as in the Yellow Emperor's Internal Classic has been discussed. With the development of traditional Chinese medicine, Chinese medicine experts summed up the contents of prevention and treatment of diseases, prevention and cure of diseases, prevention of disease and prevention of disease after illness.
\end{abstract}

\section{Treatment of Disease in the Use of Cancer Disease}

Before the tumor does not occur, through the maintenance of refined gas, can improve the body's resistance to disease, take preventive measures. In the righteousness and weakness, yin and yang and internal organs disorders, phlegm, blood stasis and other pathological products easy to produce Tumor. Through various methods nurturing righteousness, not disease prevention, so that the body blood reconcile, so as to prevent the occurrence of cancer ${ }^{[1]}$.

\section{Transfer the Spirit}

Chinese medicine believes that: people's physical and psychological changes and mental activity are closely related. Emotional disorders can cause the disease. As Yuan Dan Zhu Dan believes that breast cancer (cancer) is due to depression, boredom, sadness and other negative emotions caused by cancer is due to emotional disorders, spleen and stomach damage and other reasons, resulting in blood stagnation results. Tranquil nothingness, infuriating from the spirit, sick never. Explain the filming to maintain emotional intelligence activities, and the yin and yang Festival mood anger, through the nursed back to health, maintenance Gas, can enhance the body's anti-evil ability to prevent the occurrence of cancer ${ }^{[2]}$.

\section{Work and Rest}

Long-term lack of exercise or excessive exercise can often lead to blood stasis, tendons Pulse injury, qi and blood phlegm blockage caused by the meridian tumor. "Static to recuperate, move to rise", proper exercise can clear the meridians, reconcile the blood, improve the body's resistance to disease, prevent the occurrence of cancer $^{[3]}$.

\section{Reasonable Diet}

Personal blood from the spleen and stomach metabolism, diet section, damage the spleen and stomach is easy to get sick. if you can eat with food, release of those who demeanor, can be described as good Workers. All shows that the diet has an important impact on parthenogenesis, a reasonable diet, so that the spleen and stomach health, then the blood and viscera Sheng filling, 
which can prevent the occurrence of cancer.

\section{Treatment of Disease in the Use of Cardiovascular Disease}

Not all cardiovascular diseases require medical treatment from the very beginning. Chinese medicine has long advocated that people should be able to enhance their uprightness and thus resist evil by: moderate exercise, changing bad habits, adjusting the spirit and so on ${ }^{[4]}$.

\section{Moderate Exercise}

After the development of Ba Duan Jin, Tai Chi and other fitness methods, not only can enhance physical fitness, but also can prevent the occurrence of the disease. Appropriate exercise can make the body's blood circulation, joint slippery, emotional access, for the prevention of disease has an important role. There are studies have shown that obesity prone to high blood pressure, high blood lipids and hyperglycemia and other symptoms, and after exercise to lose weight blood pressure, blood lipids, blood sugar, etc. can have a certain degree of decline, or even can be reduced to the normal range ${ }^{[5]}$.

\section{Change Bad Habits}

"Yellow Emperor" set a few principles to avoid taboo food. In addition, a large number of studies have shown that the formation of hypertension and high sodium intake are closely related. At the same time, a large number of surveys and clinical studies have shown that excessive smoking and alcohol consumption will increase the incidence of acute myocardial infarction and sudden death. Therefore, changing bad living habits has important meanings to one's health and even life.

\section{Transfer the Spirit}

Chinese medicine has "all diseases are born of gas," "anger is on the gas, hi then slow, sad then gas consumption, fear is gas, shock is chaotic, thinking is angry," saying, emotional stimulation can lead to righteousness Weak, so that evils easy to attack the body and disease. This shows that fine God's state is an important measure of a person's health. If the mood is comfortable, then the human body is calm, the air conditioner is smooth, which is good for maintaining the stability of the blood pressure, and the prevention, occurrence and development of the disease are of great significance. Conversely, modern medicine believes that long-term stress, anger, depression and other negative emotions can cause sympathetic, autonomic, endocrine, immune and other changes that will lead to increased atherosclerosis, resulting in hypertension and other diseases. Therefore, adjusting the spirit, can enhance the human righteousness, resist evil, prevent disease, but also one of the important ways to prevent and treat hypertension.

\section{Chinese Medicine Treatment}

Danshen dripping pills have a preventive and therapeutic effect on cardiovascular diseases. Danshen dripping pills can reduce blood lipid, blood viscosity, anti-platelet aggregation and arteriosclerosis, thereby fundamentally preventing and treating coronary heart disease. Not ill "thought. In addition, some patients with coronary heart disease angina pectoris on the basis of conventional western medicine plus traditional Chinese medicine decoction can significantly improve the general symptoms of fatigue such as fatigue.

\section{Treatment of Disease Thought in the Use of Respiratory Diseases}

\section{Bronchial Asthma}

Bronchial asthma is a recurrent cough, wheezing and dyspnea, accompanied by reversible airway hyperresponsiveness, obstructive respiratory disease, is the result of a combination of mechanisms, its essence is airway chronic allergic Inflammation. Bronchial asthma are traditional Chinese medicine cough, asthma, asthma card, phlegm and other areas. Before the disease is not implemented, measures should be taken to prevent it in the first place and to control the possible influencing factors of bronchial asthma, such as environmental factors, infectious factors, genetic factors, individual factors and mental stimulation, and timely control the incidence in the embryonic stage. The main measures are: First, to keep warm to prevent exogenous evils and reduce the adverse effects of environmental factors on the disease. Pay attention to seasonal changes, suitable 
for cold and warm. Chinese medicine believes that asthma lesions organs in the lungs, spleen, and kidney three dirty. Pathological factors of asthma expectoration, cult poison and vomiting stroke each other, blocking the lung orifices, can cause cough, wheezing and other illnesses. Chinese medicine will be divided into asthma attack, Bronchial asthma through reasonable and standardized system of Chinese and Western medicine treatment, the symptoms will be significantly alleviated or even disappear, but both doctors and patients to know that the recent effective does not mean that the long-term effective, symptom relief does not mean that the cure of the disease, it should monitor the disease activity Sex, to prevent recurrence, recurrent is another major feature of bronchial asthma $^{[6]}$.

\section{Chronic Obstructive Pulmonary Disease}

Chronic obstructive pulmonary disease (COPD) is a common clinical disease ${ }^{[7]}$, frequently-occurring disease. The main symptoms are cough, sputum, asthma, characterized by recurrent, progressive increase. Due to continuous decline in lung function and lead to respiratory failure, pulmonary heart disease and lifelong disease, the need for long-term treatment, consuming a lot of manpower and financial resources, and the treatment of remission is essential, an urgent need for an economical, inexpensive, Simple, compliant method for the treatment of COPD. Chinese medicine treatment has its unique advantages, especially for relieving symptoms, reducing recurrence and improving the quality of life with a positive effect. At this stage, the research progress of traditional Chinese medicine rehabilitation in the stable period of COPD mainly focuses on the methods of health preservation, treatment both inside and outside of traditional Chinese medicine and rehabilitation of acupuncture and so on, and has achieved good curative effect. For example Oral rehabilitation for the treatment of COPD stables period, the basic recognition to Bufei, spleen, kidney-based, both Quxie. External treatment of traditional Chinese medicine, including fumigation, dipping, dressing and other methods, can significantly improve the quality of life of patients with chronic obstructive pulmonary disease, and reduce the number of winter seizures. Acupuncture rehabilitation acupuncture therapy in the stable period of COPD treatment has long been applied. In the implementation of rehabilitation, according to the principle of "slowing down the course of treatment," the main points of the treatment of lung deficiency, ointment, emphysema, Taiyuan, Zusanli and other acupoints are generally selected for patients with stable COPD.

\section{Digestive Diseases}

\section{Chronic Gastritis}

Chronic gastritis is the most common and frequent chronic digestive diseases in clinical practice. It is divided into two categories: chronic non-atrophic gastritis (superficial) and chronic atrophic gastritis (endogenous chronic gastritis) ${ }^{[8]}$. From the perspective of life and clinical observation, the occurrence of spleen and stomach diseases are mostly related to diet, emotional problems, eating disorders, emotional disorders lead to spleen and stomach dysfunction, spleen and stomach loss, liver and stomach discord, liver depression and spleen and other cards, so spleen and stomach Disease, the focus of disease prevention is not in the diet, flirting. The quality of the diet, the variety of the species and the health or not, are closely related to human health. An important feature of spleen and stomach is often recurrent with life, diet, emotional disorders, so the more the disease or stable condition, should instruct patients to pay attention to the disease after the transfer, smooth emotions, correct understanding and treatment of disease, combined with diet, Exercise and other health methods, away from the risk factors, and according to the condition need to use Yiqi spleen, liver and stomach, Yang Xin soothe the nerves and other drugs to help restore or maintain the balance of the body to prevent the recurrence of the disease or complex sense of new evil. Eating disorders, overeating, over-eating fat and sweet taste and so easy to lead to gastric stool acceptance, loss of turbidity dysfunction, stomach stagnation and the formation of evidence of stagnation, manifested as abdominal distention, vomiting acid rot.

\section{Ulcerative colitis}

Ulcerative colitis: is a non-specific inflammation, up to the rectal mucosa and submucosa, involving the rectum, sigmoid colon, and even spread to the whole colon, and was a chronic 
recurrent process. Cold wind caution adjust your diet, according to four changes in the maintenance of the spleen and stomach; cold Shushi all can hurt, but especially in the south of the Yangtze River hot and humid climate, long summer soil, the main spleen and stomach, spleen hi wet, long summer season Spleen and stomach disease, should beware of hot and humid epidemic of evil. Eat cold, spicy, greasy products, eat millet gruel or barley lotus seed porridge and other spleen and stomach, spleen and stomach strong body deserved, the body immunity and disease resistance increased, the evil can not be exclusively wounding. Is the so-called "upright inside, evil can not be done." Focus on psychological counseling, the development of stress relief channels, work and rest. Emotional tone is liver qi, liver qi are temper health, disease away from natural. Eating disorders, overeating, eating too much fat and so easy to cause fat stomach stomach satisfied, Jiangzhuo dysfunction, stomach stagnation and the formation of evidence.

\section{Blood System Diseases}

Chinese medicine Purport is idiopathic thrombocytopenic purpura (ITP), is bleeding and peripheral thrombocytopenia, the normal number of bone marrow megakaryocytes or accompanied by mature disorders as the main manifestation of the common bleeding disorders, severe cases may occur intracranial hemorrhage And life-threatening. Chinese medicine classified as "blood card" category, the first episode of ecchymosis appear on the skin, petechia, mostly juvenile pathogenesis of hemorrhagic fever, yin deficiency or hot gas does not take blood, the lesion in the myoelectricity. Chronic ITP stretches disease, chronic illness will be stasis, and late blood stasis is a more common card type. ITP patients have more evidence of yin, incense and other drugs are not too dry incense, in order to prevent catharsis of the liver. if the symptoms of liver depression more obvious, then add spleen medicine to prevent wood Wang by earth, to Xiaoyao San or Dan Zhi Xiaoyao San add to as much . For menopausal women, the use of sweet and sour jujube soup is also more common, most patients can adjust the emotional comfort. Clinically, if you see the Shenpi fatigue with poor appetite, spleen deficiency rule no doubt, "the Central Health wet, wet soil" spleen Health and wet, with or without sleepy symptoms, choose incense sand Liujunzi Tang Or Shen Ling Baizhu addition and subtraction, this is the spleen-based dialectical. If he is evidence, but also prevent the injury to the stomach and spleen and stomach, so when the time Gu Guwei, attack-free over too, $\mathrm{Zi}$ tired stagnation, Yi Qi spleen, qi and stomach, the main side of Atractylodes, Poria, Yam, Yiyiren and other spleen medicine, also added Citrus aurantium, dried tangerine peel, Amomum, wood and other gas agents. Smell less should not be more, to prevent the cart before the horse, affecting the curative effect.

\section{Conclusion}

Treatment of disease is an important part of the theory of traditional Chinese medicine, but also the most unique disease prevention and treatment of traditional Chinese medicine principles. It is necessary to uphold and carry forward the inheritance and innovation and based on the heritage to give full play to the original advantages of TCM constitution theory in treating TCM without disease, preventing and controlling chronic diseases, and modern health management. We must persist in meeting the needs of the people and people in preventing and treating traditional Chinese medicine as a top priority. Improve the Chinese medicine treatment of disease-free scientific and standardized level to contribute. Chinese medicine "to cure disease", in order to enhance the fitness as the core of the fitness, disease prevention ideas to adapt to natural changes and enhance the body's ability to fight diseases to cure the basic principles can be functional, the overall change to grasp the life, not Preventive disease, sick early treatment, disease prevention and change, illness and care.

\section{References}

[1] Meng H, Peng N, Yu M, et al. Treatment of triple-negative breast cancer with Chinese herbal 
medicine: A prospective cohort study protocol. Medicine (Baltimore). 2017. 96(44): e8408.

[2] Wang H, Tao L, Ni T, et al. Anticancer efficacy of the ethyl acetate extract from the traditional Chinese medicine herb Celastrus orbiculatus against human gastric cancer. J Ethnopharmacol. 2017. 205: 147-157.

[3] He XR, Han SY, Li XH, et al. Chinese medicine Bu-Fei decoction attenuates epithelial-mesenchymal transition of non-small cell lung cancer via inhibition of transforming growth factor $\beta 1$ signaling pathway in vitro and in vivo. J Ethnopharmacol. 2017. 204: 45-57.

[4] Wang Y, Wang Q, Li C, et al. A review of Chinese herbal medicine for the treatment of chronic heart failure. Curr Pharm Des. 2017.

[5] Yang CS, Chen G, Wu Q. Recent scientific studies of a traditional chinese medicine, tea, on prevention of chronic diseases. J Tradit Complement Med. 2014. 4(1): 17-23.

[6] Yu M, Jia HM, Cui FX, et al. The Effect of Chinese Herbal Medicine Formula mKG on Allergic Asthma by Regulating Lung and Plasma Metabolic Alternations. Int J Mol Sci. 2017. 18(3).

[7] Liao YN, Hu WL, Chen HJ, Hung YC. The Use of Chinese Herbal Medicine in the Treatment of Chronic Obstructive Pulmonary Disease (COPD). Am J Chin Med. 2017. 45(2): 225-238.

[8] Li R, Ma T, Gu J, Liang X, Li S. Imbalanced network biomarkers for traditional Chinese medicine Syndrome in gastritis patients. Sci Rep. 2013. 3: 1543. 